\title{
Recent Galactic Science Results from VERITAS on Pulsar Searches, PSR J2032+4127, and HAWC Follow-Ups
}

\author{
G. T. Richards for the VERITAS Collaboration ${ }^{* \dagger}$ \\ Georgia Institute of Technology \\ E-mail: gtrichards@gatech.edu
}

\begin{abstract}
VERITAS is a ground-based imaging atmospheric Cherenkov telescope array sensitive to very high energy (VHE, E > $100 \mathrm{GeV}$ ) gamma rays. VERITAS has detected VHE gamma-ray emission from nearly 60 astrophysical sources of varied source classes. One of the primary areas of research of VERITAS is the study of galactic particle accelerators, and among the classes of galactic objects investigated are pulsars, binary systems, and pulsar wind nebulae. In this contribution, recent results from VERITAS on the three aforementioned source classes will be presented, including: results from a search for pulsed emission from 14 young pulsars appearing in archival VERITAS data; observations of the binary system PSR J2032+4127/MT91 213, which is quickly approaching its periastron passage set to occur in 2017 November; and follow-up observations of new VHE gamma-ray sources detected by the HAWC observatory. These VERITAS observations will provide insight into the particle acceleration and radiation mechanisms at work in these galactic objects.
\end{abstract}

7th Fermi Symposium 2017

15-20 October 2017

Garmisch-Partenkirchen, Germany

* Speaker.

${ }^{\dagger}$ veritas.sao.arizona.edu 


\section{Introduction}

The galactic VHE sky contains objects of a variety of classes, including pulsar wind nebulae (PWNe), binary systems, supernova remnants, and pulsars. Many galactic gamma-ray emitters are resolved as extended sources at VHEs, which enables morphological studies and derivation of spatially dependent spectra. Studying the characteristics of the emission from these objects helps to elucidate the locations and/or mechanisms of the particle acceleration responsible for the observed radiation. Starting with Section 2, this proceeding provides an overview of the results from three recent galactic science projects from the VERITAS collaboration.

VERITAS is an array of four $12 \mathrm{~m}$ diameter IACTs and is located at the Fred Lawrence Whipple Observatory in southern Arizona ( $31^{\circ} 40^{\prime} \mathrm{N}, 110^{\circ} 57^{\prime} \mathrm{W}, 1.3 \mathrm{~km}$ a.s.1.). VERITAS started full array operations in 2007. The telescope reflectors consist of 345 hexagonal mirror facets, and the cameras comprise 499 photomultiplier tubes giving a field of view (FoV) of $\sim 3.5^{\circ}$. VERITAS is sensitive to VHE gamma-ray photons in the energy range 0.85 to $>30 \mathrm{TeV}$ with a sensitivity to detect a $1 \%$ Crab Nebula source in $\sim 25 \mathrm{hr}$. It has an angular resolution of $0.1^{\circ}$ at $68 \%$ containment and a pointing-accuracy error of less than 50 arcseconds [1]. The VERITAS data analysis results presented herein are formed using the general methodology outlined in [2].

\section{A Search for VHE Gamma Rays from 14 Pulsars Appearing in Archival VERITAS Data}

\subsection{Introduction}

Since the unexpected detection of the Crab pulsar in VHE gamma rays by VERITAS [3] and MAGIC [4, 5], one of the principal unanswered questions in VHE astrophysics has been whether or not the Crab pulsar is the sole VHE-emitting pulsar. The VHE spectrum of the Crab pulsar was recently measured to be consistent with a pure power law up to $1.5 \mathrm{TeV}$ [5] by MAGIC, which has allowed stringent constraints to be made on the mechanism and location of the particle acceleration responsible for the emission. In the time since the detection of the Crab pulsar, the Vela pulsar has been recently detected at energies up to $\sim 100 \mathrm{GeV}$ by H.E.S.S. II [6] with a $10-100 \mathrm{GeV}$ spectrum compatible with a pure power law, though the presence of curvature cannot be ruled out. Both the Crab and Vela pulsars are members of the young gamma-ray pulsar population, and both are very highly ranked in $\dot{E} / d^{2}$, taking the number one and two spots of all known gamma-ray pulsars.

VERITAS has incidentally observed the locations of many of the top northern-hemisphere pulsars according to $\dot{E} / d^{2}$ rank. Pulsars that VERITAS has observed while primarily targeting a PWN or supernova remnant (or other object with a pulsar in the same instrument FoV) are listed in Table 1, along with some properties and the VERITAS exposure time for each. There are 14 total pulsars, which are hereafter referred to as the archival pulsars. This list contains eight of the top twelve pulsars located in the northern-hemisphere sky when ranked in $\dot{E} / d^{2}$. Two of the top twelve are the Crab and Geminga pulsars, which have already been observed by VERITAS [3,7].

\subsection{VERITAS Analysis and Results}

Locations of each pulsar are taken to be those provided in the corresponding publicly available 


\begin{tabular}{ccccccc}
\hline Pulsar & R.A. $\left(^{\circ}\right)$ & Dec. $\left(^{\circ}\right)$ & $P(\mathrm{~s})$ & $\dot{P}\left(10^{-15}\right)$ & $\begin{array}{c}\dot{E} \\
\left(10^{34} \mathrm{erg} \mathrm{s}^{-1}\right)\end{array}$ & $\begin{array}{c}\text { VERITAS } \\
\text { Exposure } \\
(\mathrm{hr})\end{array}$ \\
\hline J0007+7303 & & & & & 44.8 & 32.4 \\
J0205+6449 & 31.7565 & 73.0522 & 315.9 & 357. & 2644. & 22.2 \\
J0248+6021 & 42.0776 & 64.8286 & 65.7 & 190. & 21.2 & 45.9 \\
J0357+3205 & 59.4680 & 32.0891 & 444.1 & 13.1 & 0.6 & 7.92 \\
J0631+1036 & 97.8657 & 10.6165 & 287.8 & 104. & 17.3 & 2.79 \\
J0633+0632 & 98.4339 & 6.5418 & 297.4 & 79.6 & 11.9 & 108 \\
J1907+0602 & 286.9782 & 6.0374 & 106.6 & 86.7 & 282. & 39.1 \\
J1954+2836 & 298.5798 & 28.6013 & 92.7 & 21.2 & 105. & 5.18 \\
J1958+2846 & 299.6667 & 28.7653 & 290.4 & 212. & 34.2 & 13.9 \\
J2021+3651 & 305.2726 & 36.8513 & 103.7 & 95.6 & 338. & 58.2 \\
J2021+4026 & 305.3781 & 40.4461 & 265.3 & 54.2 & 11.4 & 20.6 \\
J2032+4127 & 308.0548 & 41.4568 & 143.2 & 20.4 & 27.3 & 47.9 \\
J2229+6114 & 337.2720 & 61.2359 & 51.6 & 77.9 & 2231. & 47.2 \\
J2238+5903 & 339.6173 & 59.0624 & 162.7 & 97.0 & 88.8 & 32.5 \\
\hline
\end{tabular}

Table 1: Table listing the fourteen pulsars appearing in archival VERITAS data and some of their properties. The right ascension and declination (J2000) values given in columns 2 and 3 used in analysis are taken from the timing solutions. Columns 4 and 5 give the pulsar period $P$ and time derivative of the period $\dot{P}$. The spin-down powers $(\dot{E})$ are given in column 6 , and the final column gives the VERITAS exposure time for the pulsar. Values for $P, \dot{P}$, and $\dot{E}$ have been taken from the Fermi second pulsar catalog (2PC) [8].

Fermi-LAT timing solution ${ }^{1}$, and these coordinates are given in Table 1. The data selected for analysis satisfy two criteria: a) an archival pulsar is within $1.5^{\circ}$ of the center of the instrument FoV, and b) the date the data were taken falls within the window of validity of the corresponding Fermi-LAT pulsar timing solution used to phase-fold the VERITAS data. The data underwent a quality-selection process, with the sum of all VERITAS data analyzed here constituting a total exposure time of $483.8 \mathrm{hr}$. Additionally, a total of $7.6 \mathrm{yr}$ of Fermi-LAT data for each pulsar are analyzed with the same procedure outlined in the Fermi 2PC [8] in order to generate high-energy gamma-ray spectra above $100 \mathrm{MeV}$.

The regions in the pulsar light curves where signal and background counting are done, referred to as the "phase gates," were defined a priori . The method utilizes pulsar light curves seen in the Fermi-LAT data to define the phase gates for the VERITAS search. The method attempts to define the gates in such a way that would maximize the detection significance of the VERITAS search, assuming the locations of the peaks are the same as seen in high-energy gamma rays. Because this assumption is not necessarily true, de Jager's $H$ test [9] is also employed to probe for periodicity in the phase-folded VERITAS data.

For both the phase-gate test and the $H$ test, searches for pulsed VHE emission from all 14 archival pulsars are conducted above three energy thresholds for a total of six tests per pulsar. These six tests do not result in evidence for or the detection of VHE pulsations from any of the pulsars. For each pulsar, integral VHE flux upper limits from the VERITAS data are computed

\footnotetext{
${ }^{1}$ www.slac.stanford.edu/ kerrm/fermi_pulsar_timing/
} 
at the $95 \%$ confidence level. A phase-averaged Fermi-LAT spectrum shown with the VERITAS flux limits for the pulsar J2021+3651 is shown in Figure 1. The upper limits constrain a flux that is in many cases below the flux level of the Crab pulsar, so the broad statement can be made that potential VHE pulsed emission from each pulsar must be more faint than the VHE flux observed from the Crab pulsar ( $\sim 1 \%$ Crab Nebula level).

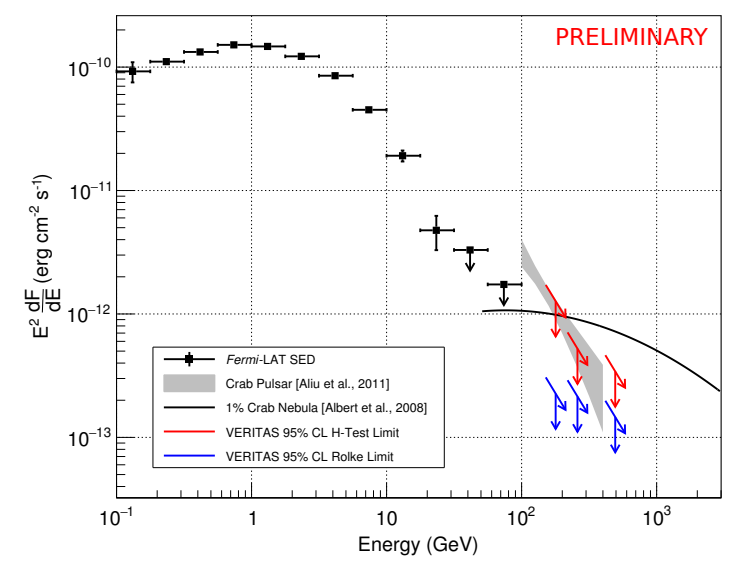

Figure 1: PSR J2021+3651 Fermi-LAT spectrum (black squares) with VERITAS flux upper limits (red and blue arrows). The Fermi-LAT spectral points are shown in black; the VERITAS 95\% CL flux ULs from the $H$ test are indicated by the red arrows; while those from the phase-gate test are given by the blue arrows. For reference, the Crab pulsar spectral bowtie from [3] is also shown (gray shaded region), in addition to the Crab Nebula spectral shape from [10] scaled to 1\% (black curved line).

Full details of the VERITAS analysis and results for all 14 archival pulsars are the subject of an upcoming publication.

\section{VERITAS Observations of the Binary System PSR J2032+4127/MT91 213}

\subsection{Introduction}

PSR J2032+4127/MT91 213 is a binary system comprising a young gamma-ray pulsar and a $15 \mathrm{M}_{\odot}$ Be star with an orbital period of $\sim 50 \mathrm{yr}$ [11]. The system will reach periastron in 2017 November, where the orbital separation will be $\sim 0.5 \mathrm{AU}$ [12]. The binary nature of the system PSR J2032+4127/MT91 213 (hereafter referred to as J2032) was only recently established in 2015 via radio observations of drastic changes to the pulsar spin-down rate [11] indicating Doppler shifting due to binary motion. Co-located with J2032 is the extended VHE gamma-ray source TeV J2032+4130, which has remained an unidentified object despite thirteen years of observations since its discovery. That TeV J2032+4130 is an extended galactic object at VHEs would typically implicate the presence of a PWN as suggested in [13], though this interpretation has been questioned due to the considerably hard VHE spectrum and the recently identified binary.

The X-ray flux from J2032 has been rising for some time, with an increase by a factor of 70 since 2002 (as of 2016 May) reported in [14]. This brightening has been interpreted as a result of 


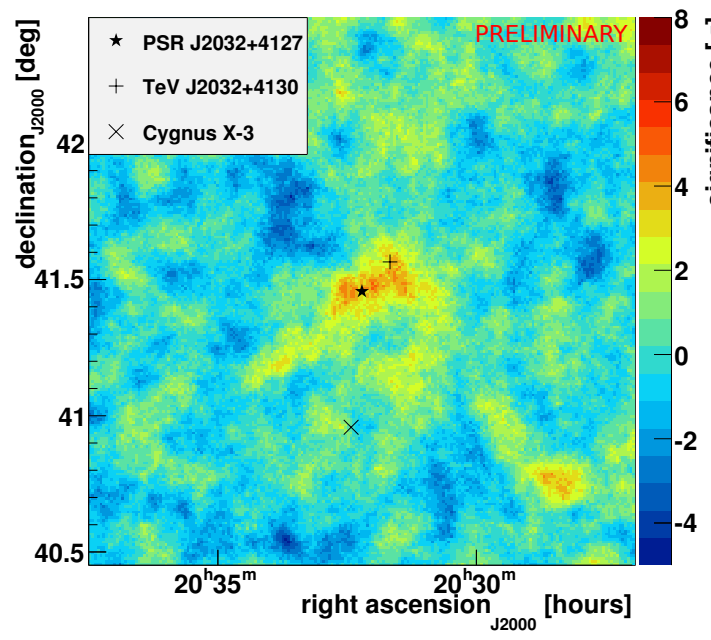

(a)



(b)

Figure 2: VERITAS significance maps of the TeV J2032+4130 region derived from data taken in 2017. In (a), a map obtained from data taken spring 2017 is shown; and shown in $(b)$ is an updated map derived only from new data taken in 2017 September. The black star indicates the position of the pulsar J2032+4127. The same color scale is used for both images.

increasing collisional wind energy in the shock region formed by the pulsar and stellar winds [14]. More recently, [15] reported a continuing X-ray flux increase up to 2017 May in Swift-XRT data. Evidence for variability in the $\mathrm{X}$-ray light curve is also present.

In contrast to the increasing X-ray flux from J2032, the high-energy gamma ray flux seen in Fermi-LAT data appears steady between 2016 May and 2017 May [15]. Further, the light curve is consistent with a uniform flux; i.e., no variability is detected.

Updated VHE gamma-ray results were recently presented by VERITAS in [15] and by VERITAS and MAGIC in a joint ATel (\#10810) published 3 October 2017 [16]. The VERITAS data in [15] comprised a total of $45 \mathrm{hr}$ with $17.6 \mathrm{hr}$ taken in fall 2012, $8.5 \mathrm{hr}$ in fall 2016 and $11.0 \mathrm{hr}$ in spring 2017. A test for an elevated flux at the pulsar location in yearly bins did not reveal any concrete evidence for an increasing flux. However, in observations conducted by VERITAS and MAGIC (15.3 hr and $5.6 \mathrm{hr}$, respectively) in fall 2017, both instruments observed a flux elevated by a factor of $\sim 2$ compared to spring/summer 2017 [16], with both VERITAS and MAGIC reporting a clear detection of a point source at the pulsar location. The source is added to the VERITAS / MAGIC catalogs as VER J2032+414 / MAGIC J2032+4127, respectively. The emergence of a point source as J2032 nears periastron confirms that the system is a gamma-ray binary, and this detection makes it just the second gamma-ray binary with a known compact companion ${ }^{2}$.

\subsection{VERITAS Analysis and Results Update}

The VERITAS results for J2032 presented herein are an update to those presented in [15], with

\footnotetext{
${ }^{2}$ The other is PSR B1259-63/LS 2883.
} 
the addition of new data taken in fall 2017 that was used in forming the results presented in ATel \#10810. The analysis methodology is the same as in [15].

In 2017 September, VERITAS accumulated a total of $15.3 \mathrm{hr}$ of new data on the J2032 location. Analysis of the September data set yields a detection significance of $6.8 \sigma$ at the location of PSR J2032+4127. The September VHE gamma-ray flux $>200 \mathrm{GeV}$ is measured to be $(4.2 \pm 0.7) \times$ $10^{-12} \mathrm{~cm}^{-2} \mathrm{~s}^{-1}$. Analysis of $\sim 21 \mathrm{hr}$ of data taken in spring 2017 gives a flux of $(2.1 \pm 0.7) \times$ $10^{-12} \mathrm{~cm}^{-2} \mathrm{~s}^{-1}$ above the same energy threshold, hence the observed flux increase is a factor of $\sim 2$. Significance maps derived from the spring and fall data sets are shown in Figure 2. A clear detection of a point source at the pulsar location can be seen in the second panel.

VERITAS will continue to monitor J2032 through periastron passage, and a detailed study is the subject of an upcoming VERITAS collaboration publication.

\section{VERITAS and Fermi-LAT Observations of New VHE Sources Discovered by HAWC}

The HAWC collaboration recently published the second HAWC source catalog (2HWC) [17], which contains 16 newly detected VHE gamma-ray sources. Of these 16, the locations of 11 appear in archival VERITAS data collected between 2007 and 2015. VERITAS has additionally observed the locations of a few of the 2HWC sources between 2015 and 2017, bringing the total observed locations to 13. The VERITAS analysis resulted in the detection of one of the new VHE sources. A Fermi-LAT analysis was also conducted in the energy range $10-900 \mathrm{GeV}$, which resulted in zero detections for both a point-source and extended-source search [18].

One region observed by VERITAS contains two of the new VHE sources in the 2HWC: 2HWC J1953+294 and 2HWC J1955+285. VERITAS has accumulated a total of $64 \mathrm{hr}$ of observations of this region, which resulted in a $5.2 \sigma$ detection of a source (VER J1952+294) coincident with 2HWC J1953+294 and a non-detection of emission from the 2HWC J1955+285 location. The likely association of 2HWC J1953+294 and VER J1952+294 is the radio PWN DA 495 [18]. The VERITAS counts map for the DA 495 region is shown in Figure 3.

The possibility of this type of multiwavelength study in gamma rays across nearly seven decades in energy underscores the synergy achieved from combined Fermi, VERITAS, and HAWC studies. Fermi and HAWC continually survey the sky, which naturally leads to new gamma-ray source detections. The higher angular resolution of an IACT such as VERITAS compared to air shower arrays allows detailed morphological studies in follow-up observations. Furthermore, spectra derived from data collected by these three instruments can be combined to produce a more complete picture of the gamma-ray radiation from a variety of sources.

For further details about VERITAS and Fermi-LAT observations of the new VHE sources detected by HAWC, please see [18] and the upcoming Fermi-VERITAS-HAWC joint publication (in preparation).

\section{Acknowledgements}

This research is supported by grants from the U.S. Department of Energy Office of Science, the U.S. National Science Foundation and the Smithsonian Institution, and by NSERC in Canada. 


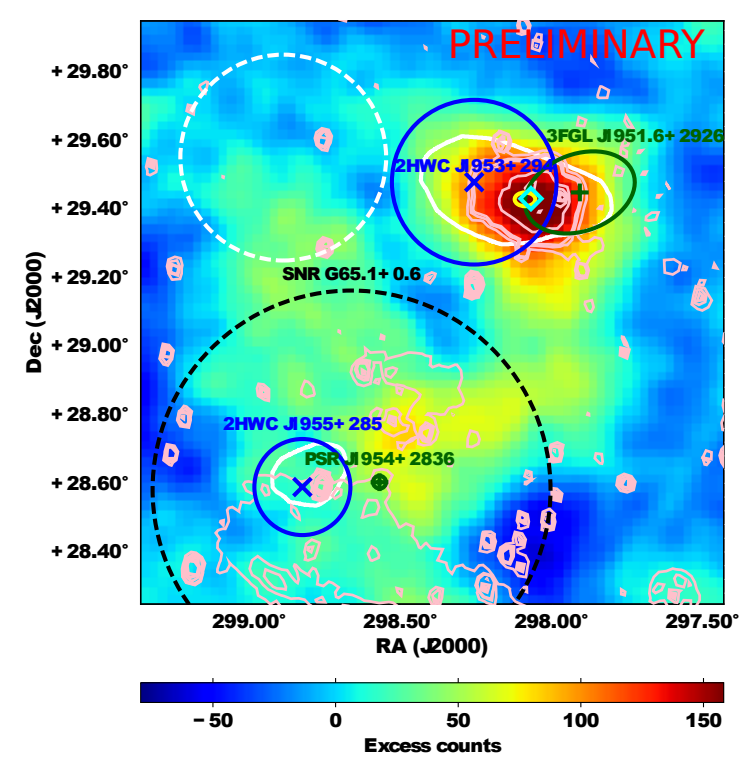

Figure 3: VERITAS VHE gamma-ray counts map of the DA 495 region. The blue circles indicate the $1 \sigma$ locations of the two $2 \mathrm{HWC}$ sources in this region, with the blue $\mathrm{x}$ marking the centroids. Sources from the Fermi third source catalog are shown in green. Radio contours for DA 495 from [19] are drawn in pink. The solid white curves indicate the $5 \sigma$ HAWC source locations. The dashed white circle shows the size of the angular cut used in the VERITAS extended source search (more details are provided in [18]).

We acknowledge the excellent work of the technical support staff at the Fred Lawrence Whipple Observatory and at the collaborating institutions in the construction and operation of the instrument.

\section{References}

[1] J. Holder, V. A. Acciari, E. Aliu, T. Arlen, M. Beilicke, W. Benbow et al., Status of the VERITAS Observatory, in American Institute of Physics Conference Series (F. A. Aharonian, W. Hofmann and F. Rieger, eds.), vol. 1085 of American Institute of Physics Conference Series, pp. 657-660, Dec., 2008, 0810.0474 , DOI.

[2] V. A. Acciari, M. Beilicke, G. Blaylock, S. M. Bradbury, J. H. Buckley, V. Bugaev et al., VERITAS Observations of the $\gamma$-Ray Binary LS I +61 303, ApJ 679 (June, 2008) 1427-1432, [0 802 .2363].

[3] VERITAS Collaboration, E. Aliu, T. Arlen, T. Aune, M. Beilicke, W. Benbow et al., Detection of Pulsed Gamma Rays Above 100 GeV from the Crab Pulsar, Science 334 (Oct., 2011) 69-, [1108.3797].

[4] E. Aliu, H. Anderhub, L. A. Antonelli, P. Antoranz, M. Backes, C. Baixeras et al., Observation of Pulsed $\gamma$-Rays Above $25 \mathrm{GeV}$ from the Crab Pulsar with MAGIC, Science 322 (Nov., 2008) 1221, [0809.2998].

[5] S. Ansoldi, L. A. Antonelli, P. Antoranz, A. Babic, P. Bangale, U. Barres de Almeida et al., Teraelectronvolt pulsed emission from the Crab Pulsar detected by MAGIC, A\& A $\mathbf{5 8 5}$ (Jan., 2016) A133, [1510.07048]. 
[6] A. Djannati-AtaÃŕ, G. Giavitto, M. Holler, B. Rudak, C. Venter and T. H. E. S. S. Collaboration, Probing vela pulsar down to 20 gev with h.e.s.s. ii observations, AIP Conference Proceedings 1792 (2017) 040028, [http://aip.scitation.org/doi/pdf/10.1063/1.4968932].

[7] E. Aliu, S. Archambault, A. Archer, T. Aune, A. Barnacka, M. Beilicke et al., A Search for Pulsations from Geminga above $100 \mathrm{GeV}$ with VERITAS, ApJ 800 (Feb., 2015) 61, [1412.4734].

[8] A. A. Abdo, M. Ajello, A. Allafort, L. Baldini, J. Ballet, G. Barbiellini et al., The Second Fermi Large Area Telescope Catalog of Gamma-Ray Pulsars, ApJS 208 (Oct., 2013) 17, [1305. 4385].

[9] O. C. de Jager, B. C. Raubenheimer and J. W. H. Swanepoel, A poweful test for weak periodic signals with unknown light curve shape in sparse data, A\& A 221 (Aug., 1989) 180-190.

[10] J. Albert, E. Aliu, H. Anderhub, P. Antoranz, A. Armada, C. Baixeras et al., VHE $\gamma$-Ray Observation of the Crab Nebula and its Pulsar with the MAGIC Telescope, ApJ 674 (Feb., 2008) 1037-1055, [0705.3244].

[11] A. G. Lyne, B. W. Stappers, M. J. Keith, P. S. Ray, M. Kerr, F. Camilo et al., The binary nature of PSR J2032+4127, MNRAS 451 (July, 2015) 581-587, [1502.01465].

[12] J. Takata, P. H. T. Tam, C. W. Ng, K. L. Li, A. K. H. Kong, C. Y. Hui et al., High-energy Emissions from the Pulsar/Be Binary System PSR J2032+4127/MT91 213, ApJ 836 (Feb., 2017) 241, [1702.04446].

[13] E. Aliu, T. Aune, B. Behera, M. Beilicke, W. Benbow, K. Berger et al., Observations of the Unidentified Gamma-Ray Source TeV J2032+4130 by VERITAS, ApJ 783 (Mar., 2014) 16, [1401.2828].

[14] W. C. G. Ho, C.-Y. Ng, A. G. Lyne, B. W. Stappers, M. J. Coe, J. P. Halpern et al., Multiwavelength monitoring and X-ray brightening of Be X-ray binary PSR J2032+4127/MT91 213 on its approach to periastron, MNRAS 464 (Jan., 2017) 1211-1219, [1609.06328].

[15] R. Bird and for the VERITAS Collaboration, PSR J2032+4127, the counterpart of TeV J2032+4130? Multiwavelength Monitoring of the Approach to Periastron, ArXiv e-prints (Aug., 2017), [1708.04718].

[16] J. Holder, TeV gamma-ray emission from PSR J2032+4127/MT91 213, The Astronomer's Telegram 10810 (Oct., 2017) .

[17] A. U. Abeysekara, A. Albert, R. Alfaro, C. Alvarez, J. D. Álvarez, R. Arceo et al., The 2HWC HAWC Observatory Gamma-Ray Catalog, ApJ 843 (July, 2017) 40, [1702 . 02992].

[18] N. Park, for the VERITAS Collaboration, Fermi-LAT Collaboration and HAWC collaboration, VERITAS and Fermi-LAT observations of TeV gamma-ray sources from the second HAWC catalog, ArXiv e-prints (Aug., 2017), [1708.05744].

[19] A. R. Taylor, S. J. Gibson, M. Peracaula, P. G. Martin, T. L. Landecker, C. M. Brunt et al., The Canadian Galactic Plane Survey, AJ 125 (June, 2003) 3145-3164. 